\title{
CORRIGENDA
}

\section{Evidence for a GVL effect following reduced-intensity allo-SCT in ALL: a British Society of Blood and Marrow Transplantation study}

PG Medd, AJ Peniket, TJ Littlewood, R Pearce, J Perry, KE Kirkland, BE Shaw, MN Potter, CF Craddock, DW Milligan, AK Fielding, DI Marks and G Cook for the British Society of Blood and Marrow Transplantation (BSBMT)

\section{Bone Marrow Transplantation (2013) 48, 1368; doi:10.1038/bmt.2013.119}

Correction to: Bone Marrow Transplantation (2013) 48, 982-987; doi:10.1038/bmt.2012.261; published online 14 January 2013

Since the publication of this article, the authors have asked that the following text be added to the acknowledgements. 'TJL and AJP were supported, in part, by the National Institute for Health Research (NIHR) Oxford Biomedical Research Centre at Oxford University Hospitals NHS Trust and University of Oxford'. The correct acknowledgements section is shown below.

The authors would like to apologize for any inconvenience this may have caused.

\section{ACKNOWLEDGEMENTS}

We wish to thank the following data managers and transplant physicians for providing data and responding to requests for further information: J Horn and D Culligan, Aberdeen Royal Infirmary; J Gribben and J Cavenagh, St Bartholomew's Hospital London;
W Clay, Birmingham Heartlands Hospital; P Breslin, University Hospitals Bristol NHS Trust; T Dalton and A Bloor, The Christie Hospital Manchester; S Kukkapalli and M Koh, St George's Hospital London; S Greep and H Hunter, Derriford Hospital Plymouth K Hussein and M Kamzi, Guy's Hospital London; B Holt and J Snowden, The Hallamshire Hospital Sheffield; F O'Boyle and J Apperley, Hammersmith Hospital London; S Hardaker and M Gilleece, Leeds Teaching Hospitals NHS Trust; R Lewin and A Hunter, The Leicester Royal Infirmary; J Ward and A Stewart, Queen Elizabeth Hospital Birmingham; S Littlewood, The Royal Marsden Hospital Sutton; J Sudak and S Mackinnon, The Royal Free Hospital London; L McNally and G Jackson, The Royal Victoria Infirmary Newcastle; K Thompson, University College Hospital London and A Robertson and P Johnson, The Western General Hospital Edinburgh. TJL and AJP were supported, in part, by the National Institute for Health Research (NIHR) Oxford Biomedical Research Centre at Oxford University Hospitals NHS Trust and University of Oxford.

Author contributions: PM designed the study, collected data and wrote the manuscript, AP and TL designed the study, performed transplants and contributed data, JP and KK ran the data registry and collected data, RP performed statistical analysis, BS, MP, AF, DM, CC, DM and GC performed transplants and contributed data, all authors critically reviewed and approved the manuscript.

\section{Pre-transplant risk factors for cryptogenic organizing pneumonia/bronchiolitis obliterans organizing pneumonia after hematopoietic cell transplantation}

\begin{abstract}
H Nakasone ${ }^{1,2,16}$, M Onizuka ${ }^{3,16}$, N Suzuki ${ }^{4,16}$, N Fujii ${ }^{5,16}$, S Taniguchi ${ }^{6}$, K Kakihana ${ }^{7}$, H Ogawa $^{8}$, K Miyamura $^{9}$, T Eto $^{10}$, H Sakamaki $^{7}$,

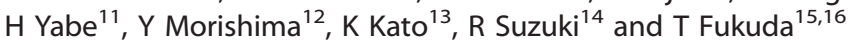

${ }^{1}$ Division of Bone and Marrow Transplantation, Stanford University School of Medicine, Stanford, CA, USA; ${ }^{2}$ Division of Hematology, Saitama Medical Center, Jichi Medical University, Saitama, Japan; ${ }^{3}$ Department of Hematology, Tokai University School of Medicine, Isehara, Japan; ${ }^{4}$ Department of Pediatrics, Sapporo Medical University, Sapporo, Japan; ${ }^{5}$ Department of Hematology and Oncology, Okayama University Graduate School of Medicine, Dentistry and Pharmaceutical Sciences, Okayama, Japan; ${ }^{6}$ Department of Hematology, Toranomon Hospital, Tokyo, Japan; ${ }^{7}$ Hematology Division, Tokyo Metropolitan Cancer and Infectious Disease Center Komagome Hospital, Tokyo, Japan; ${ }^{8}$ Division of Hematology, Department of Internal Medicine, Hyogo College of Medicine, Hyogo, Japan; ${ }^{9}$ Department of Hematology, Japanese Red Cross Nagoya First Hospital, Nagoya, Japan; ${ }^{10}$ Department of Hematology, Hamanomachi Hospital, Fukuoka, Japan; ${ }^{11}$ Department of Cell Transplantation and Regenerative Medicine, Tokai University School of Medicine, Isehara, Japan; ${ }^{12}$ Division of Epidemiology and Prevention, Aichi Cancer Center Research Institute, Nagoya, Japan; ${ }^{13}$ Department of Pediatric Hematology and Oncology, Children's Medical Center, Japanese Red Cross Nagoya First Hospital, Nagoya, Japan; ${ }^{14}$ Department of Hematopoietic Stem Cell Transplantation Data Management and Biostatics, Nagoya University School of Medicine, Nagoya, Japan; ${ }^{15}$ Stem Cell Transplantation Division, National Cancer Center Hospital, Tokyo, Japan and ${ }^{16}$ Working Group for Complications after HCT of the Japan Society for Hematopoietic Cell Transplantation
\end{abstract}

Bone Marrow Transplantation (2013) 48, 1368; doi:10.1038/bmt.2013.153

Correction to: Bone Marrow Transplantation (2013) 48, 13171323; doi:10.1038/bmt.2013.116; published online 12 August 2013

Since the publication of this issue, the author have noticed that Hiroyasu Ogawa was omitted from the authors list. This error has now been rectified. The correct authors and affiliation lists are shown above and the corrected article appears in this issue.

The authors would like to apologize for any inconvenience this may have caused. 